\title{
Challenges in Diagnosis, Treatment and Follow-up of Patients Presenting with Central Nervous System Infections in a Resource-Limited Setting
}

\author{
Aleksandra Leligdowicz*, Michael Katwere, Theresa Piloya, Allan Ronald, \\ Andrew Kambugu, Elly Katabira
}

\begin{abstract}
Central Nervous System (CNS) infections are associated with significant mortality and morbidity. Accurate diagnosis is necessary for prompt treatment and increased chances of survival. However, there are many challenges to correct diagnoses in resource-limited settings, including the HIV epidemic, late presentation of symptomatic individuals, limited availability of laboratory diagnostic tests as well as treatment, and inadequate access to funds accompanied by lack of financial support from developed countries. This article presents case reports of patients admitted to the Mulago Hospital in Kampala, Uganda that exemplify challenging diagnoses of tuberculous meningitis (TBM), cryptococcal meningitis (CM), toxoplasmosis, and primary CNS lymphoma (PCNSL). Also included is a literature review of the pathology, diagnosis, and treatment of TBM, CM, toxoplasmosis, and PCNSL in immunocompromised patients.
\end{abstract}

KEY WORDS: Uganda, HIV, CNS infections, Tuberculous Meningitis, Cryptococcal Meningitis, Toxoplasmosis, Primary CNS Lymphoma

\section{INTRODUCTION}

Central Nervous System (CNS) infections carry a high morbidity and mortality rate. They affect any part of the CNS (cerebral parenchyma, meninges, spinal cord) and can be caused by infectious organisms such as bacteria, fungi, parasites, viruses, and prions resulting in inflammation. Accurate diagnosis and prompt treatment of many CNS infections is difficult even in the most sophisticated medical centers. In resource-limited settings seen in developing countries, the rapid diagnosis that is essential for effective treatment is even more challenging.

The Human Immunodeficiency Virus (HIV) is the

*To whom correspondence should be addressed: Aleksandra Leligdowicz, McGill University Faculty of Medicine, 3655 Promenade Sir William Osler, Montreal,Canada, H3G 1Y6 causative agent of the Acquired Immunodeficiency Syndrome (AIDS). At the end of 2003, an estimated 34.6 - 42.3 million people worldwide were living with HIV, of whom two thirds live in Africa $(1,2)$. The term AIDS was first used in 1981 to describe a combination of opportunistic infections and tumours occurring in young homosexual men who were dying from infections that a healthy immune system would easily combat (3). Today, the pandemic spread of HIV and lack of antiretroviral therapy distribution in developing countries is contributing to AIDS-related opportunistic infections, including those affecting the central nervous system.

The profile of CNS infections in Sub-Saharan Africa has changed as a result of the HIV epidemic. For example, a study of 284 patients hospitalized with meningitis in Johannesburg, South Africa, found tuberculous meningitis (TBM) to be the most 
common diagnosis (25.4\%), followed by acute bacterial meningitis $(22.5 \%)$, viral meningitis $(14.1 \%)$, and cryptococcal meningitis (13\%) with more than $40 \%$ in-hospital mortality (4). In a study of 200 patients diagnosed with meningitis in Harare, Zimbabwe, cryptococcal meningitis was the most common diagnosis $(45 \%)$, followed by mononuclear meningitis $(27 \%)$, acute bacterial meningitis (16\%), and TBM (12\%); with in-hospital mortality also greater than $40 \%$ (5). These high in-patient CNS infection-related mortality rates in resource-limited settings call for the medical community to address the management of CNS infections. In the past, simple algorithmic approaches have led to improved patient outcomes for life threatening infections such as pneumonia in HIV-1 patients (6). It is important to design a similar efficient algorithmic approach for the timely diagnosis and treatment of CNS infections, especially in the face of the growing HIV/AIDS epidemic, in settings where access to sophisticated diagnostic tools is not readily available.

Uganda is a country located in East Africa, with a population of 26.4 million (7). After independence was gained from Britain in 1962, the oppressive dictatorial regimes of Idi Amin and Milton Obote, as well as the poverty and the onset of HIV/AIDS epidemic, took an enormous toll on Ugandan society $(8,9)$. In 1986, Museveni became the president, and by the 1990 's, the stability in the country started being regained. However, despite a relatively stable political state at the macro level, widespread intrinsic poverty continues at the microeconomic level, especially in the rural areas, where the majority of the population lives. Today, more than 50\% of the population is attempting to survive on less than $1 \$ \mathrm{US}$ per day and AIDS is the leading cause of death in the 15-49 age group (8). The health sector in Uganda is underfunded and the accessibility to health care services is inadequate with 18,700 people per doctor and 870 people per hospital bed (10). This situation amounts to a heavy burden of preventable diseases and a low life expectancy [45 years in 2002 (7)]. Uganda, however, has been repeatedly reported as one of the only African countries where HIV/AIDS prevalence is decreasing, with a fall in the national prevalence from $13 \%$ in the early 1990 s to $4.1 \%$ by the end of 2003 (1).

Mulago Hospital is a national referral and teaching hospital in Kampala, the capital city of Uganda. The hospital general medical wards admit on average 3050 patients a day, and overcrowding is a problem. Accessbility of essential drugs and supplies is highly variable. The most common medical conditions on the general wards include AIDS-related infections and the most common causes of death include CNS infections, pneumonias, and tuberculosis (11).

The Academic Alliance for AIDS Care and Prevention in Africa (AAACP) is a not-for-profit organization formed in June 2001 by a group of physicians and scientists from Makerere University in Kampala, Uganda and the Infectious Disease Society of America with HIV/AIDS care experts from North America (12). The goal of the AAACP is to fight Africa's biggest health threat, HIV/AIDS, and to improve the quality of medical care for the 27 million HIV-infected people living in sub-Saharan Africa (13). The organization supports the Infectious Disease Center (IDC) at the Mulago Hospital and is involved in many ongoing studies funded by the Bill $\&$ Melinda Gates Foundation. The aim of one such study is to design an algorithmic approach that maximizes available resources to enhance the outcome of patients presenting with CNS symptoms. The study's goal is to improve the accuracy and speed of diagnosis and thereby lead to more prompt diseasespecific treatment and a decrease in the in-patient hospital stay when compared to the current standard of care. Four case reports of patients from this study are presented in this article, followed by a review of the pathology, diagnosis, and treatment of TBM, CM, toxoplasmosis, and PCNSL in immunocompromised patients.

\section{LITERATURE REVIEW}

Opportunistic infections are common complications of advanced immunodeficiency in individuals with HIV-1 infection. Following involvement of the lung, the central nervous system is the second most commonly affected organ (23). Neurological disease is the first manifestation in $10-20 \%$ of symptomatic HIV-infected individuals, presenting in $39-70 \%$ of HIV-infected patients and found at autopsy in up to $79 \%$ of AIDS patients $(20,21,23,24,26,27)$. Neurological complications associated with HIV-1 infection may be primary (HIVdementia, inflammatory demyelinating polyneuropathy, cerebrovascular disease) or secondary (Tuberculosis, T. gondii, Cryptococcus neoformans, or Nocardia infections, Primary CNS Lymphoma) (20).

The diagnostic approach to an immunocompromised host with CNS infection depends on the analysis of the patient's clinical manifestations of CNS disease, including the acuteness or sub-acuteness of the disease. Most patients with CNS infections may be grouped into those with meningeal signs, or those with mass lesions. Meningitis and encephalitis tend to present more acutely whereas CNS mass lesions tend to 
present subacutely or chronically. Meningitis of bacterial, cryptococcal, or tuberculous origin can be diagnosed from stain, culture, or serology of the CSF. In contrast, patients with CNS mass lesions usually require a tissue biopsy to arrive at a specific etiologic diagnosis (28), making it a very challenging diagnosis in a resource-limited setting.

\section{1) Tuberculous Meningitis (TBM)}

Approximately 2 billion people in the world are infected with tuberculosis, yet the risk of developing clinical tuberculosis (TB) post-infection is only $10 \%$ in an immunocompetent individual; However, HIV coinfection increases this risk over 3 times (15). Central nervous system TB occurs in $2 \%$ of all patients with TB and in $10 \%$ of those with AIDS, and can be the first manifestation of AIDS in $20 \%$ of HIV positive patients $(29,30)$. The total number of TB cases is increasing. Tuberculosis is caused by infection with Mycobacterium tuberculosis, through droplet inhalation. Infection is localized within alveolar macrophages and causes a brief but significant bacteremia, seeding bacteria to other organs, including the meninges or brain parenchyma, forming sub-ependymal foci called Rich foci. During tuberculous meningitis (TBM) development, a Rich focus ruptures into the subarachnoid space, resulting in a local $\mathrm{T}$-lymphocyte dependant immune response that heralds the onset of meningitis (15). Clinical findings include fever $(54 \%)$, headache $(50 \%)$, altered mental status (42\%), cough (33.3\%), nausea/vomiting $(21 \%)$, meningismus (17\%), and seizures (4\%) (30). Diagnosis is dependent on lumbar puncture (LP) and CSF analysis (the gold standard). However, culture is insensitive and very slow (taking up to 6-8 weeks) to aid clinical decisions $(6,30)$. The search for acid fast bacilli (AFB) by direct Ziehl-Neelsen staining of the CSF represents the best rapid laboratory diagnostic technique (16), however, this lacks sensitivity as AFBs are seen in only $10-20 \%$ of patients with TBM $(15,16)$. Lymphocytosis is between 100-1000 cells $/ \mathrm{mm}^{3}$, with raised CSF protein and reduced CSF glucose in $70 \%$ of patients (15). CT and MRI imaging of the brain is significant for meningeal enhancement, communicating hydrocephalus, infarcts in territory of middle cerebral artery, edema, and tuberculomas (solitary or multiple), with the basal ganglia most often affected (30). Treatment consists of four-drug combination chemotherapy including isoniazid, rifampicin, pyrazinamide, and ethambutol or streptomycin for the first two months $(15,30)$. The duration of the continuation phase varies between 1022 months $(15,31)$ and includes rifampicin and isoniazid only or all four drugs (15). A corticosteroid such as dexamethasone can be added as an adjuvant as it may reduce the effects of inflammation, long term neurological sequelae, and mortality (32).

Clinical manifestations of TBM are variable, but are not altered when comparing HIV-infected patients to non-infected individuals (29). The key challenge of TBM diagnosis is distinguishing it from bacterial meningitis. CFS cell counts, glucose and protein results can be similar in partially treated bacterial meningitis and early TBM, making a clear diagnosis often impossible (15). Patients with TBM, however, present with a longer history that can include cranial nerve palsies; clear CSF with a moderate number of lymphocytes and neutrophils with an increased protein concentration; a low ratio of CSF: blood glucose; CT scan consistent with TBM; a chest radiograph that can be consistent with active pulmonary TB; and good response to antituberculosis chemotherapy, providing additional support for the diagnosis of tuberculosis (16).

Among HIV-infected patients, TBM mortality approaches $80 \%$ (30). Rapid diagnosis and treatment can prevent death and neurological morbidity. However, current laboratory methods are insensitive and slow, and new techniques, such as polymerase chain reaction (PCR), are not suitable for widespread use in the developing world. In addition, multiple drug resistant strains of $\mathrm{M}$. tuberculosis are emerging, making therapy a further challenge in the treatment of this disease in any setting.

\section{2) Cryptococcal Meningitis (CM)}

Of the systemic fungal infections in HIV-1 infected patients, cryptococcal meningitis (CM) is the most common and its incidence among AIDS patients varies between $10 \%$ in the United States and Europe and $30 \%$ in sub-Saharan Africa $(24,33)$. In Uganda, advanced HIV-related immunosuppression is the strongest independent risk factor for cryptococcal disease, and in HIV-infected adults it is the leading contributor to death (34). CM is caused by infection with the saprophytic yeast Cryptococcus neoformans, an encapsulated fungus acquired from the environment via inhalation, affecting patients with CD4 T-lymphocyte counts of less than $100 \times 10^{6}$ cells/L (24). Pathology of CM reveals basilar, nonexudative chronic meningeal inflammation as well as cryptococcomas and microabscesses in the basal ganglia region. In immunocompetent patients, $\mathrm{CM}$ presents with neurologic manifestations without evidence of systemic infection, most commonly presenting with meningismus and photophobia (24). In HIV-positive patients however, presentation includes non-specific signs and symptoms such as 
fever (58\%), headache (32\%), cough (23\%), meningismus $(13 \%)$, depressed level of consciousness (5\%), and confusion (4\%) (34). In addition, elevated intracranial pressure (ICP) occurs in over $50 \%$ of HIV-infected patients with CM without accompanying hydrocephalus or cerebral edema. Diagnosis is based on detection of the cryptococcal capsular polysaccharide antigen $(\mathrm{CrAg})$ in the $\mathrm{CSF}$ and serum, CSF India Ink stains, and culture of the organism in CSF (which is considered to be the definitive diagnosis of CM) $(21,24)$. Treatment is of utmost importance as mortality from cryptococcal disease is inevitable in the absence of appropriate therapy. Treatment includes induction with intravenous Amphoteracin B combined with 5flucytosine for two weeks, followed by consolidation therapy with fluconazole for 8 weeks (24). In patients with elevated ICP, serial lumbar punctures are indicated. If refractory to serial LPs, less conservative measures such as lumbar drains and lumbar peritoneal shunts can be employed to normalize the ICP (25). There is limited resistance associated with fluconazole use and it may therefore be used for lifelong secondary prophylaxis to prevent re-infection with different strains of C. neoformans (33). In addition, exposure to fluconazole for more than 90 days proved to reduce the risk of CM by $82 \%$ and it can therefore be used for primary prophylaxis when CD4 T-lymphocyte count falls below $200 \times 10^{6}$ cells/L (34). However, in an African setting, where access to appropriate laboratory facilities and funds is limited, CD4 T-lymphocyte levels are of little value. CM is a distressing and painful illness that is compounded by rudimentary palliative care and difficulties in prescribing opiate analgesia in much of Africa. In addition, the absence of antiviral therapy further adds to the challenges of preventing and fighting the disease. Thus, in an African setting a cryptococcal vaccine would be the most relevant approach to the prevention of CM.

\section{3) Cerebral Toxoplasmosis}

Cerebral toxoplasmosis is the most common cerebral mass lesion in HIV-1 infected patients (20, 24, 36). It is caused by infection of an intracellular parasite, T. gondii, whose difinitive host is the cat. As cats excrete oocysts, the oocysts sporulate and become infectious tissue cysts. Humans can acquire the parasite, leading to latent infection. It is estimated that $10-40 \%$ of HIV-1 infected patients are seropositive for anti-Toxoplasma antibodies and 25$50 \%$ of patients experience symptomatic infection in the absence of antimicrobial prophylaxis (24). Cerebral toxoplasmosis is caused by reactivation of latent infection as a result of progressive loss of cellular immunity, developing when CD4+ Tlymphocyte count falls below $100 \times 10^{6}$ cells/L (20, 24). Reactivation causes multifocal necrotizing encephalitis and surrounding inflammation, most commonly in the frontal and parietal areas of the brain, especially in the cortico-medullary junction, the basal ganglia, and the thalamus, involving both the gray and/or white matter $(20,24)$. Disease in symptomatic patients manifests by headache, confusion, fever, lethargy, seizures, cranial nerve palasies, psychomotor changes, hemiparesis and/or ataxia. Diagnosis can be made based on the presence of anti-toxoplasma antibodies in the blood (although not specific) or in the CSF. In contrast, a more specific (although not very sensitive) diagnostic method is PCR amplification of T. gondii-DNA (24). Standard tests of CSF specimens in patients with toxoplasmosis are nonspecific and are often normal (20). Computed Tomography (CT) and MRI imaging findings include multiple ring-enhancing lesions [although $27 \%$ of patients have a single lesion on imaging studies (22)] with surrounding edema and mass effect, with MRI imaging being more sensitive (37). A definite diagnosis requires demonstration of tachyzoites in a biopsy specimen of the brain (20). Treatment of toxoplasmosis consists of sulfadiazine in combination with pyrimethamine with added folinic acid supplementation to counter the hematopoietic toxicity of pyrimethamine. If sulfonamides cannot be tolerated, a combination of clindamycin and pyrimethamine can be used. Response to anti-toxoplasmosis therapy observed within the first 2-3 weeks of treatment is also used for diagnostic purposes $(21,28)$. Induction therapy is given for 6 week, followed by maintenance therapy with lower doses of the same regimen (20). Primary prophylaxis with trimethoprim-sulfamethoxazole is recommended for $\mathrm{T}$. gondii seropositive patients with CD4+ T-lymphocyte counts of $100 \times 10^{6}$ cells/L or less (24). However, despite increasing prescription of primary prophylaxis (which is not always successful), it is likely that toxoplasmosis will remain an important source of neurologic morbidity in AIDS patients, and continue to be a presenting illness in previously unidentified HIV-infected individuals (36).

\section{4) Primary CNS lymphoma (PCNSL)}

Primary CNS lymphoma (PCNSL) is the second most common cause of AIDS-related focal brain lesions after cerebral toxoplasmosis $(20,24)$. It is an extranodal, non-Hodgkin B-cell type neoplasm that is 1000-3900 times more common in HIV-1 infected patients than in the general population, especially in 
patients with CD4 T-lymphocyte counts below 50 $\times 10^{6}$ cells/L (24, 38). Furthermore, survival of patients with AIDS-associated PCNSL is significantly less than in non-AIDS-associated cases (2.6 vs 18.9 months) (39). Almost 100\% of AIDS-related PCNSL contain Epstein-Barr virus (EBV) genomic DNA within the lymphoma cells, an uncommon finding in tumours from immunocompetent patients (39). EBV infects B-cells and causes clonal expansion of infected cells. Normally, regulation of B-cell growth is mediated by T-lymphocytes. Therefore, quantitative abnormalities in T-lymphocytes in HIV-1 infected patients may account for uncontrolled EBV-induced B-cell proliferation and subsequent neoplastic transformation in an immunologically privileged site, such as the CNS (39). The clinical presentation of PCNSL in patients with AIDS includes an approximately two-month history of altered mental status (48-60\%), seizures (15-41\%), focal neurologic deficits or sensory findings (31-78\%), headache and evidence of increased intracranial pressure (5-45\%), as well as manifestations of AIDS such as opportunistic infections $(20,24)$. Neuroimaging findings (CT or MRI) may reveal solitary or multifocal hypodense enhancing lesions (in a ring or a homogeneous pattern) exhibiting mass effect with surrounding edema $(20,24)$. A majority of the tumours are located supratentorially and adjacent to CSF pathways (in the corpus collosum, cerebrum, basal ganglia, and occasionally in the brain stem) (20). CSF analysis is rarely helpful but it is used for excluding other diagnoses. However, PCR amplification of the CSF for EBV DNA may serve as a tumor marker for PCNSL and it is the standard of care in patients with CT findings of focal enhancing CNS-lesions (20). The most commonly used treatment modality is external beam radiation therapy, and standard whole brain radiation (4000-6000 cGy) should be used (24). However, it is important to note that this treatment modality can lead to long-term cognitive dysfunction as a result of progressive cerebral demyelination (39). Radiotherapy in patients with non-AIDS-associated PCNSL can be supplemented with steroid chemotherapy, which works on the principle of its lymphotoxic effect (39). Surgical resection is usually not possible due to the fact that the tumour is most often multifocal and resection does not improve prognosis (20). It is important to note that empiric anti-toxoplasmosis therapy is the first-line treatment approach to most HIV-1 positive patients with focal brain lesions and should thus be employed in patients presenting with PCNSL-related pathology prior to administration of PCNSL-specific treatment (24). Remission of AIDS- related PCNSL has been described in association with highly active antiretroviral therapy (HAART) (40). Unfortunately, antiretroviral therapy is not widely available in many areas of the world most affected by the burden of HIV infection, and its availability and use in developing countries will have a crucial influence on the incidence and prognosis of diseases such as PCNSL.

\section{5) TBM vs Toxoplasmosis vs PCNSL}

The diagnosis of neurological diseases is not always evident as it is often very difficult to distinguish, especially between toxoplasmosis and PCNSL. In patients with mass effect on CT scans, the diagnosis can be aided by examining anti-Toxoplasma antibodies (toxoplasmosis probability is 0.87 if seropositive and probability of PCNSL is 0.74 if seronegative). PCR amplification of the CSF for T. gondii-DNA or EBV-DNA increases the probability of toxoplasmosis or PCNSL to 0.96 if the test is positive (41). Furthermore, when the differential diagnosis of a CNS mass lesion based on neuroradiologic characteristics is between TBM, toxoplasmosis, and PCNSL, a trial of empiric therapy is warranted (28). Antitoxoplasmosis therapy may be initiated and usually results in clinical improvement after 2-3 weeks of therapy. Nonresponse to antitoxoplasmosis therapy would justify an empiric trial of antituberculous therapy. Lack of response to anti-Toxoplasma and antituberculous therapy should suggest a noninfectious etiology, such as primary CNS lymphoma, for which treatment in a resourcepoor setting is limited, especially in a setting where access to antiretroviral therapy is available to a minority of AIDS patients.

\section{PATIENT CASE PRESENTATIONS}

\section{1) A Socially-Challenged Case - Algorithm Arm}

$\mathrm{KE}$, a 23-year-old male prisoner, was referred to Mulago Hospital for specialized treatment after failure to improve during 3 days of management at the Murchison Bay Prison Referral Hospital where he was admitted with a history of new onset of left upper limb weakness. He had a past history of pulmonary tuberculosis and was on the continuation phase of anti-TB treatment. His present history included two weeks of a mild headache, convulsions, confusion, and limb weakness that was most pronounced in the left upper limb. On admission, he had healed scars suggestive of past generalized maculo-papular rash and oral candidiasis. He was afebrile, had no nausea or vomiting, and had an unremarkable cardiorespiratory physical exam. His Glasgow Coma Scale (GCS) was $15 / 15$, Karnofsky score was $70 \%$, neck was supple, 
Kerning's sign was negative, and papilledema was absent. His motor exam was significant for left upper limb monoplagia (power of 0/5), generalized brisk reflexes, right flexor plantar response, unresponsive left plantar response, and a normal sensory exam. Clinical studies were negative for serum Cryptococcal antigen (sCrAg), and a rapid HIV test was positive. A lumbar puncture (LP) was not performed due to focal neurological signs. The presumptive diagnosis was cerebral toxoplasmosis with a differential diagnosis of bacterial meningitis and tuberculous meningitis (TBM) secondary to history of tuberculosis with uncertain compliance to treatment. He was started on empiric treatment for toxoplasmosis (1440 mg Septrin po $\mathrm{q} 8 \mathrm{~h}$ ), bacterial meningitis ( $\mathrm{g}$ IV ceftriaxone QD), continued oral anti-TB treatment (Isoniazid and Ethambutol), and phenytoin (300 mg po QD). The patient's condition progressively declined and on the 5th day after admission, he rapidly deteriorated clinically with a GCS of $7 / 15$, slowing respiration, and right upper limb held in decorticate position. At that time, the ward team performed an LP. Cerebrospinal fluid (CSF) analysis revealed 10 cells/cm3, protein $200 \mathrm{mg} / \mathrm{dL}$, Gram and ZiehlNeelsen (ZN) stains were negative as was the bacterial culture. A CT scan was done on the same day and showed an extensive ill-defined, non-enhancing hypodense lesion involving most of the right parietal lobe with global sulcial effacement, right lateral ventricle compression with $0.9 \mathrm{~cm}$ mid-line shift to the left, all suggesting an inflammatory cerebral lesion with massive brain edema with imminent danger of infratentorial coning (figure 1). Based on the LP and CT results, diagnoses included toxoplasmosis, TBM, and cerebral brain abscess.

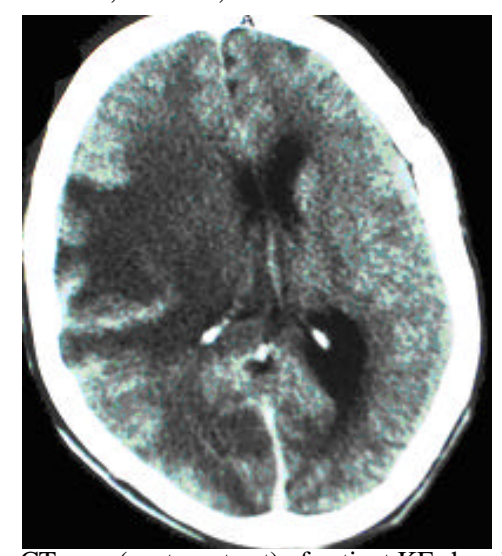

Figure 1. CT scan (post-contrast) of patient KE showing an extensive ill-defined, non-enhancing hypodense lesion involving most of the right parietal lobe with global sulcial effacement, right lateral ventricle compression with $0.9 \mathrm{~cm}$ mid-line shift. Findings are suggestive of an inflammatory cerebral lesion with massive brain edema with imminent danger of infratentorial coning. Possible differential diagnoses include toxoplasmosis, TBM, and cerebral brain abscess.
Management was modified to include a corticosteroid (8mg IV dexamethasone $\mathrm{q} 8 \mathrm{~h}$ ), a diuretic (40mg IV furosemide $\mathrm{q} 12 \mathrm{~h}$ ), and the insertion of a nasogastric tube. Despite continued treatment for the presumed differential diagnoses, the patient continued to deteriorate and died on the 15th day after admission.

The case demonstrates several challenges unique to a resource-limited setting. At the Mulago hospital, patients are cared for by relatives, who feed the patient and provide basic nursing care, including bathing, changing, and turning the patient. For the prisoner, social support was poor as he received limited care from prison staff. For example, upon deterioration, the patient could no longer accept solid food. The prison changed the food to porridge, but its supply was not regular. Also, the basis for the original diagnosis of pulmonary TB was not certain and the patient's compliance was unknown.

In conclusion, the patient presented with progressively worsening signs of focal neurological deficits. He was managed for toxoplasmosis, TBM, and a bacterial cerebral abscess. The neurological clinical and diagnostic findings were suggestive of a progressive cerebral lesion that was unresponsive to treatment and complicated by limited social support, leading to death on the 15th day after admission.

\section{2) A complicated diagnosis - Algorithm Arm}

NR, a 32-year-old female, was brought into the Mulago Hospital by her neighbours who found her "unconscious" in her house. They reported that she complained of feeling "unwell" for the past 3 days. On admission, she was found to be semicomatose with a fever of $38.4^{\circ} \mathrm{C}$, stertorous respiration, healed scars suggestive of maculo-papular rash (unknown serostatus), GCS was 11/15, neck was stiff, Kerning's sign indeterminable, generalized brisk reflexes, normal muscle bulk, and no papilledema. Her cardiorespiratory exam was normal. Upon arrival to the casualty department, she was managed for cerebral malaria $(600 \mathrm{mg}$ IV quinine in a $500 \mathrm{ml} 5 \%$ dextrose solution). She was then transferred to the emergency room, where the working diagnosis was bacterial meningitis. IV quinine was stopped and IV ceftriaxone (2g QD) was started. An LP was done within 24 hrs of admission. Results were obtained on the second day of admission and showed 800 cells $/ \mathrm{cm}^{3}$ (predominately lymphocytes), protein 80 $\mathrm{mg} / \mathrm{dL}$, negative India ink, Gram stain, and ZN stain, and no bacterial growth on overnight culture. Based on these findings, the working diagnosis changed to tuberculous meningitis. Oral anti-TB drugs were started (two months intensive phase of $800 \mathrm{mg}$ Ethambutol, $450 \mathrm{mg}$ Rifampicin, $1.2 \mathrm{~g}$ Pyrazinamide, 
$300 \mathrm{mg}$ Isoniazid QD) and ceftriaxone was stopped. Patient improved over the subsequent 7 days with a GCS of 15/15. However, despite improvement, the patient complained of continued mild headache and had a spiking fever and therefore remained under hospital care. On the 15th day after admission, she started deteriorating; she had a worsening headache, fluctuating mental status, papilledema, and no focal neurological signs. An LP was repeated despite increased intracranial pressure (in the setting of Cryptococcal meningitis, lumbar punctures are not contraindicated and therapeutic LPs are often done despite increased intracranial pressure - see discussion). The analysis revealed turbid CSF, 250 cells $/ \mathrm{cm}^{3}$ (predominately lymphocytes), protein 120 $\mathrm{mg} / \mathrm{dL}$, Gram stain 2+ encapsulated yeast cells, 1+ gram negative rods, 2+ leukocytes, India ink $2+$ for encapsulated yeast cells, negative ZN stain, culture isolated 2+ Cryptococcus neoformans. The diagnosis changed to mixed Cryptococcal meningitis (CM) and gram-negative bacterial meningitis. Treatment was modified to IV Amphoteracin B (50 mg QD) for 14 days, IV ceftriaxone (2g QD) for 10 days. After the $4^{\text {th }}$ day of treatment, the patient dramatically improved and was discharged after the completion of Amphoteracin B treatment, 36 days after admission.

The case demonstrates many findings common in Uganda. Firstly, it illustrates an example of the most common pathology in patients presenting with fever, plasmodium falciparum infection (malaria). The case also shows the diagnostic challenges associated with the initial improvement response observed with antiTB treatment which is then followed by deterioration as is demonstrated by the altered LP results. Similar improvement of symptoms after anti-tuberculosis treatment has been reported, with the reason for improvement is not understood (14). Additionally, the case is an example of clinical diagnosis of HIV using the criteria of a major opportunistic infection (CM). The patient received voluntary counseling and was referred to the infectious disease clinic after discharge. It is important to note that if the patient had been in the standard of care arm of the study, as opposed to the algorithmic arm, a repeated LP would not have been performed because the patient initially improved with anti-TB drugs. In the algorithm arm, patient deterioration calls for a repeated LP.

\section{3) Tuberculous Meningitis - Algorithm Arm}

NM, a 35-year-old female, presented to the Mulago Hospital with a 14-day history of gradual onset of headache and a 6-month history of nuchal pain, photophobia, recurrent high-grade fevers, malaise, and no past history of treatment for opportunistic infections. The 6-month history of poor health concerned NM's relatives and prompted them to do an HIV test without NM's consent or awareness 3 weeks prior to admission, the results of which were positive. On admission, she was delirious, her GCS was 15/15, her Karnofsky score was 60\%, Kernig's sign was positive, and her neck was stiff. Papilledema could not be assessed because the patient was delirious and resistant. The physical exam revealed generalized hyperreflexia $(3+)$ with normal power, tone, and sensation in all 4 limbs, and bilaterally down-going toes. The remainder of the physical exam was unrevealing. Based on the findings, the working diagnosis was acute bacterial meningitis (ABM). An LP was done within 24 hours of admission and the patient was started on empirical IV ceftriaxone $(2 \mathrm{~g}$ QD). On the second day of admission, the patient deteriorated clinically with the development of aphasia and a GCS of 9/15. She continued being managed for ABM until the third day of admission when LP results were obtained. The results showed a white blood cell count of 250 cells $/ \mathrm{ml}^{3}$ (predominantly lymphocytes), protein of $120 \mathrm{mg} / \mathrm{dL}$, India ink and Gram-stain were negative, and the $\mathrm{ZN}$ stain was significant for $1+$ acid fast bacilli (AFB), 2+ WBC, with cultures to follow. The diagnosis changed to tuberculous meningitis (TBM) and she was started on a 2-month induction treatment for tuberculosis consisting of a 4-drug regimen (Isoniazid, Ethambutol, Rifampicin, Pyrazinamide [HERZ]) and IV dexamethasone ( $8 \mathrm{mg} \mathrm{q} 8 \mathrm{~h}$ ), and the ceftriaxone was stopped. On the fifth day of admission, the patient deteriorated further and developed respiratory distress. High flow $0_{2}$ was added to the patient's treatment, but the patient rapidly deteriorated and died the same day. The likely cause of death was uncal-tonsilar herniation due to raised intracranial pressure.

The difficulty in interpreting CSF results is not unique to this case. CSF results can be very similar in partially treated bacterial meningitis and early TBM and making a clear diagnosis is often extremely challenging (15). Delays in diagnosis and treatment are major contributing factors to the high mortality and, as a result, approximately $30 \%$ of patients with TBM die despite anti-tuberculosis chemotherapy (16). Even though anti-TB treatment in Uganda is free, the free drugs at the Mulago Hospital are dispensed only two times per week, meaning that patients presenting on non-dispensing days must purchase their own medication. Since NM did not present on a dispensing day, she had to buy the initial 3 doses of treatment. In Uganda, most drugs are not supplied by the hospital and it is upon the patient to purchase their own drugs, 
making hospital stay affordable only to those with money.

\section{4) Toxoplasmosis or Primary CNS Lymphoma?}

DD, a 45 year-old male, presented to the Mulago Hospital with complaints of a $2 \frac{1 / 2}{2}$ month history of recurrent fevers, a one month history of cough and "not speaking", and a 3 day history of "failure to walk" and "urinary incontinence". Due to his aphasia, his history was obtained from his wife. He was generally healthy and this was his first admission to the hospital. For the first month of his fevers, he received treatment for malaria at various private clinics. However, the persistent fevers and lack of improvement despite treatment with antimalarial drugs prompted his wife to urge him to get tested from HIV. An HIV test was done at Mulago hospital one month prior to admission. Upon finding out that he was HIV+, he refused to share this information with his wife. However, after repeated pleas, he revealed his status and never spoke from that moment on. He refused to feed, became withdrawn, and kept himself isolated during the one month before admission. Three days prior to admission, he stopped walking due to lower limb weakness, developed urinary incontinence and constipation, all of which prompted his wife to bring him to the Mulago Hospital casualty department on the 6th of September.

On presentation, the physical exam was difficult to interpret because of DD's psychiatric presentation. He appeared to be mildly wasted, was fully conscious with a GCS of 15/15, with intact comprehension but with expressive aphasia. He had oral candidiasis, his neck was "stiff", Kernig's sign was negative, and papilledema was not assessed. The power in his right and left upper limb were $5 / 5$ and $4 / 5$ respectively and $3 / 5$ in both lower limbs. The tone and reflexes were normal in the upper limbs. There was hypertonia and hyperreflexia $(3+)$ in both lower limbs. Toes were down-going bilaterally and the remainder of the physical exam was unremarkable. He was diagnosed with depression and subjective paraparesis with a differential diagnosis of organic brain syndrome secondary to opportunistic infections associated with HIV (TBM, toxoplasmosis, CM, ABM, encephalitis). To rule out organic brain syndrome, a lumbar puncture was done and results revealed clear and colourless CSF that was under moderate pressure, a white blood cell count $<5$ cells $/ \mathrm{mm}^{3}$, protein of 80 $\mathrm{mg} / \mathrm{dL}$, and Gram stain, India ink, ZN, VDRL, TPHA, and culture were all negative. The LP was therefore undiagnostic with only mild protein elevation. His CBC revealed lymphocytopenia corresponding to immunosuppression associated with HIV/AIDS.
Chest $\mathrm{x}$-ray and liver function tests were normal. The working diagnosis remained depression and he was treated with Fluoxetine (20 mg po QD).

On the 4th day of admission, weakness progressed and became right-sided hemiparesis. Toxoplasmosis titers were done on the same day and came back negative (IgG $4.1 \mathrm{ng} / \mathrm{ml}$ [ $>5 \mathrm{ng} / \mathrm{ml}$ being positive], IgM index 0.37 [ $>0.96$ being positive]). On the 7 th day of admission, weakness further progressed and became right-sided hemiplegia. A CT scan of the head was done on the same day and revealed a nodular, ring-enhancing lesion in the anterior part of the left basal ganglia with brain edema and moderately raised intracranial pressure. There was a mid-line shift of 0.5 $\mathrm{mm}$ to the right with lateral ventricle compression on the left. The findings were suggestive of an inflammatory process with abscess formation and a high suspicion of toxoplasmosis. The diagnosis postCT scan was cerebral toxoplasmosis with differential diagnoses of primary CNS lymphoma and bacterial abscess (mycotic aneurysm, tuberculoma, metastatic lesion less likely). The patient was started on high dose Septrin (1920 mg po q6h) for toxoplasmosis and 2 days of high dose IV steroids ( $8 \mathrm{mg}$ dexamethasone $\mathrm{q} 8 \mathrm{~h}$ ) followed by oral prednisolone (40 $\mathrm{mg}$ QD) for brain edema. After four days of anti-toxoplasmosis and steroid treatment, the patient remained in hospital and the wife reported slight improvement. However, the patient remained aphasic with right-sided weakness.

The case would be challenging in any setting. Firstly, it illustrates the difficulty associated with

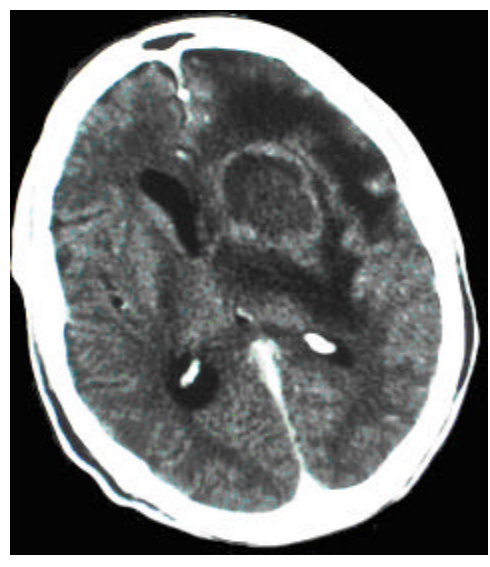

Figure 2. CT scan (post-contrast) of patient DD showing a nodular, ring-enhancing lesion in the anterior part of the left basal ganglia with accompanying brain edema and moderately raised intracranial pressure. There is a $0.5 \mathrm{~mm}$ mid-line shift with lateral ventricle compression on the left. The findings are suggestive of an inflammatory process with abscess formation and a high probability of toxoplasmosis with differential diagnoses of primary CNS lymphoma and a bacterial abscess (less likely mycotic aneurysm, tuberculoma, or metastatic lesion). 
acceptance of serostatus due to stigmatization associated with being HIV positive. HIV status challenges relationships. In this case, the husband feared revealing his status to his wife for fear of his wife leaving him alone with his illness. The impact of HIV infection on the disruption of families through separation, divorce, and widowhood has been examined among women in the Rakai district, Uganda (17). The research results revealed that family dissolution is more common among HIV-infected women and that infected women in HIV-discordant couples (where only one partner is positive) are especially vulnerable to separation or divorce. It is very likely that DD was facing similar fears, especially because his wife was HIV negative.

The case also demonstrates challenges in diagnosis of psychiatric illness versus an organic process. The patient's depressive state on admission was diagnosed as a psychiatric illness and would have been overlooked as such had further investigations not been performed. Finally, the fact that a CT scan revealed a brain lesion is only somewhat helpful. The fact that anti-toxoplasmosis antibody titers were negative [they are positive in most patients either before or at time of diagnosis (18) in part because the tests used for measuring anti-toxoplasmosis antibody titers have a high sensitivity and thus are positive in most of suspected cases (19), being negative in <3-6\% of patients with toxoplasmosis (20)] and the CT scan revealed a single lesion [toxoplasmosis usually presents with multiple ring-enhancing lesions (21)] questions the value of toxoplasmosis treatment. There is, however, one report of anti-Toxoplasma antibody negative cases with pathologically proven disease (in $22 \%$ of patients) and single enhancing lesions on CT (in $27 \%$ of patients) (22), providing further evidence of the complexity of diagnosing CNS infections in any setting, irrespective of available diagnostic modalities. It is important to note that if the patient's lesion is a primary CNS lymphoma, treatment in a resource-poor setting is limited (see discussion for challenges in differentiating toxoplasmosis and primary CNS lymphoma). At the time of this report, the patient remains in the hospital awaiting response to treatment.

\section{CONCLUSION}

Postmortem studies have shown that there is a high frequency of neuropathologic abnormalities and that there often may be mixed pathology in HIV-1 infected patients (27). This suggests that the CNS is a frequent target for HIV-related pathology as well as that diagnosis is often challenging and complicated. Diagnosis may be difficult because findings of lumbar puncture, CT scan, and MRI are relatively nonspecific, making diagnosis complicated in even the most sophisticated centres (20). In resource-poor settings, the limited availability and high associated cost of diagnostic techniques such as CT scans, MRI, and PCR analysis of the CSF, as well as risks associated with brain biopsy, further limit the probability of obtaining an accurate diagnosis, thereby preventing prompt and specific treatment. Therefore, an algorithmic approach, such as the one currently being studied, to help in overcoming these challenges may result in better treatment and outcome as well as shorten in-hospital stays in areas where resources are limited.

\section{ACKNOWLEDGEMENTS}

The authors would like to thank Dr. Merle Sande and the staff of the Adult Infectious Disease Center at the Mulago hospital, the McGill International Health Initiative for the OMAF (Osler Medical Aid Foundation) Travel scholarship for an elective in a developing nation and the Bill and Melinda Gates Foundation for financial and material support.

\section{REFERENCES}

1. UNAIDS. 2004 Report on the Global HIV/AIDS Epidemic: 4th Global Report. Geneva: December 2004.

2. Steinbrook R. The AIDS epidemic in 2004. N Engl J Med 2004;351(2):115-7.

3. Rowland-Jones SL. Timeline: AIDS pathogenesis: what have two decades of HIV research taught us? Nat Rev Immunol 2003;3(4):343-8.

4. Bergemann A, Karstaedt AS. The spectrum of meningitis in a population with high prevalence of HIV disease. QJM 1996;89(7):499-504.

5. Hakim JG, Gangaidzo IT, Heyderman RS, Mielke J, Mushangi E, Taziwa A, Robertson VJ, Musvaire P, Mason PR. Impact of HIV infection on meningitis in Harare, Zimbabwe: a prospective study of 406 predominantly adult patients. AIDS 2000;14(10):1401-7.

6. Viale P, Petrosillo N, Castelli F, Cadeo B, Carosi G. Algorithm-based management of pneumonia in HIV-1infected patients. Lancet 2002;359(9303):359-60.

7. CIA World Factbook. www.immigrationusa.com/wfb2004/uganda/ uganda people.html. 2004.

8. Wakhweya A, Kateregga C, Konde-Lule J, Mukyala R, Sabin L, Williams M, Heggenhougen K. Orphans and Their Households: Caring for Their Future - Today: The Government of Uganda Ministry of Gender, Labour, and Social Development; 2002.

9. Judas T. Uganda: The Secret War. The New York Review 2004;51(14):62-64.

10. Ministry of Health - Uganda. Statistical Abstract. April 2003.

11. Mulago Hospital Complex. www.mulago.or.ug. Kampala.

12. Quinn TC. The academic alliance for AIDS care and prevention in Africa. Hopkins HIV Rep 2001;13(6):1-4.

13. Academic Alliance for AIDS Prevention and Care in Africa. www.aaacp.org. 
14. Zahra LV, Azzopardi CM, Scott G. Case report Cryptococcal meningitis in two apparently immunocompetent Maltese patients. Mycoses 2004; 47(3-4): 168.

15. Thwaites G, Chau TT, Mai NT, Drobniewski F, McAdam K, Farrar J. Tuberculous meningitis. J Neurol Neurosurg Psychiatry 2000;68(3):289-99.

16. Thwaites GE, Chau TT, Stepniewska K, Phu NH, Chuong LV, Sinh DX, White NJ, Parry CM, Farrar JJ. Diagnosis of adult tuberculous meningitis by use of clinical and laboratory features. Lancet 2002;360(9342):1287-92.

17. Porter L, Hao L, Bishai D, Serwadda D, Wawer MJ, Lutalo T, Gray R. HIV status and union dissolution in sub-Saharan Africa: the case of Rakai, Uganda. Demography 2004;41(3):465-82.

18. Renold C, Sugar A, Chave JP, Perrin L, Delavelle J, Pizzolato G, Burkhard P, Gabriel V, Hirschel B. Toxoplasma encephalitis in patients with the acquired immunodeficiency syndrome. Medicine (Baltimore) 1992;71(4):224-39.

19. Wilson M, Remington JS, Clavet C, Varney G, Press C, Ware D. Evaluation of six commercial kits for detection of human immunoglobulin $\mathrm{M}$ antibodies to Toxoplasma gondii. The FDA Toxoplasmosis Ad Hoc Working Group. J Clin Microbiol 1997;35(12):3112-5.

20. Skiest DJ. Focal neurological disease in patients with acquired immunodeficiency syndrome. Clin Infect Dis 2002;34(1):103-15.

21. Collazos J. Opportunistic infections of the CNS in patients with AIDS: diagnosis and management. CNS Drugs 2003;17(12):869-87.

22. Porter SB, Sande MA. Toxoplasmosis of the central nervous system in the acquired immunodeficiency syndrome. N Engl J Med 1992;327(23):1643-8.

23. Masliah E, DeTeresa RM, Mallory ME, Hansen LA. Changes in pathological findings at autopsy in AIDS cases for the last 15 years. Aids 2000;14(1):69-74.

24. Mamidi A, DeSimone JA, Pomerantz RJ. Central nervous system infections in individuals with HIV-1 infection. J Neurovirol 2002;8(3):158-67.

25. Fessler RD, Sobel J, Guyot L, Crane L, Vazquez J, Szuba MJ, Diaz FG. Management of elevated intracranial pressure in patients with Cryptococcal meningitis. J Acquir Immune Defic Syndr Hum Retrovirol 1998;17(2):137-42.

26. Levy RM, Bredesen DE, Rosenblum ML. Neurologic complications of HIV infection. Am Fam Physician 1990;41(2):517-36.

27. Burns DK, Risser RC, White CL, 3rd. The neuropathology of human immunodeficiency virus infection. The Dallas, Texas, experience. Arch Pathol Lab Med 1991;115(11):1112-24.

28. Cunha BA. Central nervous system infections in the compromised host: a diagnostic approach. Infect Dis Clin North Am 2001;15(2):567-90.

29. Berenguer J, Moreno S, Laguna F, Vicente T, Adrados M, Ortega A, Gonzalez-LaHoz J, Bouza E. Tuberculous meningitis in patients infected with the human immunodeficiency virus. N Engl J Med 1992;326(10):668-72.

30. Whiteman M, Espinoza L, Post MJ, Bell MD, Falcone S. Central nervous system tuberculosis in HIV-infected patients: clinical and radiographic findings. AJNR Am J Neuroradiol 1995;16(6):1319-27.

31. Phuapradit $P$, Vejjajiva A. Treatment of tuberculous meningitis: role of short-course chemotherapy. Q J Med 1987;62(239):249-58.

32. Girgis NI, Farid Z, Kilpatrick ME, Sultan Y, Mikhail IA. Dexamethasone adjunctive treatment for tuberculous meningitis. Pediatr Infect Dis J 1991;10(3):179-83.

33. Pfaller M, Zhang J, Messer S, Tumberland M, Mbidde E, Jessup C, Ghannoum M. Molecular epidemiology and antifungal susceptibility of Cryptococcus neoformans isolates from Ugandan AIDS patients. Diagn Microbiol Infect Dis 1998;32(3):191-9.

34. French N, Gray K, Watera C, Nakiyingi J, Lugada E, Moore M, Lalloo D, Whitworth JA, Gilks CF. Cryptococcal infection in a cohort of HIV-1-infected Ugandan adults. Aids 2002;16(7):1031-8.

35. Oursler KA, Moore RD, Chaisson RE. Risk factors for cryptococcal meningitis in HIV-infected patients. AIDS Res Hum Retroviruses 1999;15(7):625-31.

36. Cohen BA. Neurologic manifestations of toxoplasmosis in AIDS. Semin Neurol 1999;19(2):201-11.

37. Levy RM, Rothholtz V. HIV-1-related neurologic disorders. Neurosurgical implications. Neuroimaging Clin N Am 1997;7(3):527-59.

38. Raez L, Patel P, Feun L, Restrepo A, Raub W, Cassileth P. Natural history and prognostic factors for survival in patients with acquired immune deficiency syndrome (AIDS)-related primary central nervous system lymphoma (PCNSL). Crit Rev Oncol 1998;9:199-208.

39. Fine HA, Mayer RJ. Primary central nervous system lymphoma. Ann Intern Med 1993;119(11):1093-104.

40. McGowan J, Shah S. Long term remission of AIDS-related primary central nervous system lymphoma associated with highly active antiretroviral therapy in patients with AIDS. AIDS 1998;12:952-953.

41. Antinori A, Ammassari A, De Luca A, Cingolani A, Murri R, Scoppettuolo G, Fortini M, Tartaglione T, Larocca LM, Zannoni G and others. Diagnosis of AIDS-related focal brain lesions: a decision-making analysis based on clinical and neuroradiologic characteristics combined with polymerase chain reaction assays in CSF. Neurology 1997;48(3):687-94.

Aleksandra Leligdowicz is a 3rd year medical student at McGill University on a leave-of-absence doing a PhD degree in Clinical Medicine at Oxford University on a Rhodes Scholarship. She is currently working at the Medical Research Council Laboratories in the Gambia and Projecto Saude de Bandim in Caio, a remote village in Guinea Bissau, where she is focusing her research on the host immune response to HIV-2 infection in West Africa. 